\title{
Wiki Trans : une communauté de savoir sur la transidentité
}

Wiki Trans: a Knowledge Community on Transidentity

Wiki Trans : Eine Wissensgemeinschaft zum Thema Transidentität

\section{Bérengère Stassin}

\section{OpenEdition}

1 Journals

\section{Édition électronique}

URL : http://journals.openedition.org/ctd/3723

DOI : $10.4000 /$ ctd. 3723

ISSN : 2491-1437

\section{Éditeur}

Chaire Unesco Pratiques émergentes en technologies et communication pour le développement

\section{Édition imprimée}

ISBN : 2491-1437

\section{Référence électronique}

Bérengère Stassin, «Wiki Trans : une communauté de savoir sur la transidentité », Communication, technologies et développement [En ligne], 9 | 2021, mis en ligne le 29 mars 2021, consulté le 31 mars 2021. URL : http://journals.openedition.org/ctd/3723 ; DOI : https://doi.org/10.4000/ctd.3723

Ce document a été généré automatiquement le 31 mars 2021.

Communication, technologies et développement 


\title{
Wiki Trans : une communauté de savoir sur la transidentité
}

\author{
Wiki Trans: a Knowledge Community on Transidentity \\ Wiki Trans : Eine Wissensgemeinschaft zum Thema Transidentität
}

Bérengère Stassin

\section{Introduction}

1 Les personnes transgenres ne se reconnaissent pas dans le genre qui leur a été assigné à la naissance en fonction de leur sexe biologique, à la différence des personnes cisgenres pour qui genre assigné et caractéristiques sexuelles coïncident. Pour lutter contre la dysphorie que suscite la conviction intime et constante d'appartenir au genre opposé, les personnes trans entament la plupart du temps un parcours de «transition ». Cette transition est sociale (coming out, changement de prénom et de pronom, changement d'état civil), mais peut aussi être médicale. Dans ce cas, elle consiste en la prise d'un traitement hormonal substitutif féminisant (MtoF) ou masculinisant (FtoM) et en la réalisation d'opérations chirurgicales. Ces dernières ont généralement vocation à féminiser ou masculiniser les corps, mais ne sont pas systématiquement des opérations de réattribution sexuelle contrairement aux idées reçues qui circulent dans l'imaginaire collectif. Changer de genre, ou plus précisément affirmer son genre, ne signifie pas nécessairement changer de sexe.

Le parcours de transition est marqué par de nombreuses interrogations. Si c'est un parcours psychique, social, administratif et médical, c'est aussi un parcours que l'on peut qualifier d'« informationnel » et de «documentaire». En effet, les personnes en questionnement ou en transition ont une importante activité de recherche d'information, qui s'exerce principalement aujourd'hui au sein du web. Ce dernier est devenu une source d'information incontournable, regorgeant de ressources et de témoignages publiés sur les médias sociaux, mais offrant aussi un accès à de l'information médicale et scientifique grâce aux archives ouvertes et aux revues en accès libre. Il favorise en outre le développement de communautés en ligne dont les 
membres formalisent, à travers leurs échanges, des connaissances tacites et développent des savoirs. "Quand tu fais une transition, tu obtiens un PhD en sociologie, en droit et en médecine", nous confie, lors d'un entretien, Menica Folden, la fondatrice du site Wiki Trans auquel nous avons consacré l'étude présentée dans cet article. Par ce propos, elle souligne un aspect essentiel du parcours de transition : l'acquisition d'un savoir expérientiel.

3 Les savoirs développés et remédiés par et pour les trans favorisent l'autonomisation des parcours et l'émancipation face aux instances médicales et au système hospitalier jugés pathologisants, psychiatrisants et infantilisants. Il ne s'agit bien sûr pas de prôner l'automédication ou de nier le fait que certaines personnes aient besoin d'un suivi psychologique durant une ou plusieurs étapes du parcours, mais d'offrir des repères et de partager des informations permettant de faire les bons choix et de connaitre ses droits. Par exemple, le processus peut parfois être retardé par des endocrinologues refusant de délivrer un traitement hormonal sans diagnostic référentiel d'une dysphorie de genre ou, pour le dire autrement, sans attestation psychiatrique, alors que cela n'est pourtant plus obligatoire en France : «[l]'acquisition d'une expertise médicale profane et la remédiation des savoirs scientifiques en santé au sein de la communauté vise davantage à garantir un suivi se déroulant dans les meilleures conditions, qu'à s'affranchir totalement du cadre médical classique » (Delias, Lallet, 2018).

Si ce rôle de remédiation des savoirs et de vulgarisation de l'information médicale a longtemps été joué par les associations trans militantes, on voit de plus en plus émerger au sein de Twitter, de YouTube et d'Instagram des personnalités influentes et des regroupements d'individus qui s'engagent à leur tour dans une activité de documentation du parcours médical, social et administratif, mais aussi dans une activité de "pédagogie » à destination des personnes cisgenres afin de leur permettre de comprendre et de soutenir un proche qui s'engage dans une transition, de dépasser les stéréotypes et préjugés qui constituent le terrain fertile de la transphobie. C'est donc dans ce nouveau contexte et dans ce nouveau paysage informationnel que Wiki Trans émerge en 2018, à l'initiative de Menica Folden, une femme transgenre.

5 En consultant la page d'accueil du site, on constate que parmi les rubriques qui le composent l'une s'intitule «Pour la transition» et l'autre «Pour les alliés ». On peut aussi lire le message suivant : «Hey! Nous sommes le Wiki Trans. Toutes les ressources pour les personnes trans en questionnement ou en transition, leurs proches et leurs alliés ». On comprend d'emblée que ses destinataires sont à la fois les personnes transgenres et les personnes cisgenres et que la remédiation des savoirs par les trans se fait ici pour les trans et pour les cis. En entrant dans la rubrique «Pour la transition ", on découvre différentes sous-rubriques: "en questionnement», "coming out», «traitements hormonaux ", «médical ", «administratif», etc. Le site met donc à disposition d'un public hétérogène des « ressources » ayant trait à différents aspects de la transidentité et de la transition. Se pose alors la question de la nature et de la fonction de ces ressources, de leur visée communicationnelle en fonction du public ciblé. En outre, la présence du terme "wiki » dans le nom du site renvoie à l'écriture collaborative et à la mise en commun d'expertises et de connaissances. L'expression « Nous sommes le Wiki Trans» confirme la présence d'un groupe d'individus réunis autour d'un intérêt commun et engagés dans la production d'un corpus de "ressources", de documents et discours. Cela pose aussi la question du profil des contributeur.tricess ainsi que des modalités de participation et de contribution qui leur 
sont offertes pour mener à bien le projet qui les rassemble. Ainsi, cet article ne propose pas une étude de réception du site, mais une analyse de son projet infocommunicationnel et de ses logiques de fonctionnement.

\section{Les pratiques informationnelles des personnes transgenres}

\section{Parcours de transition et besoin informationnel}

6 En Sciences de l'information et de la communication, le concept de «pratiques informationnelles» est employé pour désigner ce que les usagers font avec l'information, mais aussi pour désigner le processus qu'ils mettent en place pour satisfaire leurs besoins informationnels. Il peut être défini comme la « manière dont un ensemble de dispositifs, de sources formelles ou non, d'outils, de compétences cognitives sont effectivement mobilisés, par un individu ou un groupe d'individus, dans les différentes situations de production, de recherche, d'organisation, de traitement, d'usage, de partage et de communication de l'information " (Chaudiron, Ihadjadene ; 2010 : 16). Parmi les « situations » qui suscitent l'intérêt des chercheurs de la discipline depuis quelques années, en France, mais surtout à l'étranger, se trouvent celles des personnes transgenres engagées dans un processus de transition sociale et parfois médicale. Les personnes qui s'engagent dans un changement d'état civil, dans un traitement hormonal, dans des actes de chirurgie, le font généralement de manière réfléchie et cherchent des réponses aux nombreuses questions qu'elles se posent avant de prendre une décision : comment faire ? Vers qui me tourner? Comment en parler à mes proches? Quels sont les effets et les coûts des traitements médicaux? Tous les médecins sont-ils aptes à accueillir mon projet avec bienveillance ? La chirurgie est-elle un passage obligatoire pour vivre au mieux ma transidentité ? Le parcours de transition suscite donc un fort besoin informationnel.

7 Comme tout besoin, le besoin informationnel correspond à une sensation de manque. Il s'agit plus particulièrement d'un manque d'information, de la reconnaissance d'une lacune dans son état de connaissance (Le Coadic, 2004), d'une incertitude qui empêche d'agir (Saracevic, 1996) et qui provoque inconfort et anxiété (Kuhlthau, 1991). La prise de conscience de ce manque de connaissance pour résoudre un problème ou accomplir une tâche va alors conduire l'individu vers une démarche intentionnelle de recherche d'information. Mais une recherche d'information ne se fait pas sans effort. Elle implique en effet que l'individu fournisse des efforts physiques (se déplacer jusqu'à une bibliothèque, une librairie, un ordinateur situé dans autre pièce), mais surtout intellectuels et psychologiques afin d'être en mesure de sélectionner de l'information, de relier les informations entre elles, de raisonner à partir d'elles et, dans certains cas, d'être prêt à lire des informations désagréables ou choquantes (Choo, 2006 ; Simonnot, 2006), en l'occurrence pour les personnes transgenres des informations ancrées dans les discours de haine et les discours transphobes, des articles psychologisants et pathologisants, des images d'agression ou des images pornographiques (Adams, Peirce, 2006 ; Augustaitis et al., 2021).

8 Les chercheuses suédoises Pohjanen et Terttu (2016) ont montré que les besoins informationnels changent à mesure que les personnes avancent dans leur parcours. Une première étape consiste à rechercher de l'information qui sera plutôt liée à la 
transidentité, pour comprendre le phénomène, mettre des mots sur un ressenti, une dysphorie, identifier des pairs, trouver des modèles pour "visualiser» ce qu'est physiquement une transition et des conseils sur la manière de faire un coming out, une transition sociale. Une seconde étape est davantage en lien avec la transition médicale et la recherche d'informations relatives aux traitements hormonaux, aux opérations chirurgicales et à leurs risques, ou encore aux possibilités de traitement à l'étranger. Ces chercheuses ont identifié des barrières cognitives auxquelles les personnes ont pu se retrouver confrontées au cours de leurs recherches d'information et qui sont dues à un manque de ressources documentaires en langue maternelle (le suédois) ou au manque de vocabulaire en début de parcours (comment faire une recherche sur la transidentité si on ne connaît pas le terme ?). D'autres barrières ont été mises en avant par la chercheuse américaine Madeline Deutsch (2016) comme la difficulté pour certaines personnes de comprendre de l'information médicale ou scientifique non vulgarisée.

\section{L'accès à l'information médicale en ligne}

Une étude consacrée aux pratiques informationnelles des personnes trans, conduite en 2006 par Adams et Peirce, a identifié différentes sources d'informations auxquelles elles avaient recours : quelques films et émissions de télévision, de la littérature, des groupes de parole et de soutien, mais surtout l'Internet et le naissant web 2.0. Ces derniers sont, selon les personnes interrogées à l'époque, très prometteurs pour la communauté. Ils offrent en effet la possibilité de faire des recherches d'information secrètement et de manière totalement anonyme, ce que permet moins facilement de faire un emprunt en bibliothèque ou un achat en librairie. Les forums de discussion et les blogs favorisent aussi l'émergence de groupes de parole en ligne permettant de tisser des liens, de se sentir moins seul et d'être rassuré quant à la possibilité d'un avenir. Dix ans plus tard, d'autres études consacrées au sujet vont mettre en exergue le rôle désormais central des médias sociaux (Twitter, Facebook, Tumblr, Instagram, Reddit, Discord), des forums et des sites d'association dans les parcours de transition: ils apportent de nombreuses réponses sur le parcours social et administratif, mais regorgent surtout d'informations médicales relatives aux traitements hormonaux et chirurgicaux, mais aussi à la santé mentale et au bien-être (Augustaitis et al., 2021 ; Hawkins, Giesking. 2017 ; Hawkins, Haimson 2018 ; Karami et al., 2018 ; Lallet, Delias, 2018).

Le recours à Internet et aux médias sociaux pour accéder à de l'information dans le domaine de la santé n'est pas le propre des personnes trans. Dans les années 2000, de nombreuses communautés de patientes émergent autour de ces dispositifs (Legros, 2009). Grâce au mouvement de l'open access et des archives ouvertes, l'information scientifique non vulgarisée, produite par des spécialistes à destination de spécialistes, est désormais accessible au grand public. Il circule également en ligne de l'information vulgarisée par les médecins eux-mêmes, des médecins-blogueurs adeptes de la médecine 2.0 (Dupagne, 2011), mais aussi par des amateures de science disposant d'un capital culturel suffisamment élevé pour comprendre le jargon médical ou encore par des patientes (ou leurs proches) devenues expertes de la maladie qui les affecte. Les médias sociaux vont donc permettre de combler des besoins informationnels en offrant des ressources hybrides relevant tantôt de l'information médicale ou spécialisée, tantôt du témoignage et de la mise en commun des vécus (Paganelli, Clavier, 2011 ; Paganelli et al., 2014). 
11 Il émerge alors au sein du web des savoirs «expérientiels » et une figure du "patient expert» dont l'expertise et la légitimité sont fondées sur un parcours de vie et une expérience. Ces savoirs reposent sur des compétences thérapeutiques relatives aux médicaments et sur des compétences psychosociales développées pour vivre avec un traitement qui peut parfois être lourd. Leur élaboration est favorisée par l'expérience quotidienne de la maladie ainsi que par les échanges entre pairs (Jouet, Las Vergnas, 2010). À travers ces échanges les personnes formalisent des connaissances tacites et développent des savoir-faire et des savoir-être liés au problème qui les affecte ou à l'intérêt commun qui les réunit, constituant alors des communautés de pratique (Wenger, 1998). Bien que ces communautés soient avant tout tournées vers la formalisation de connaissances tacites, il n'est pas rare que leurs membres partagent ou génèrent des connaissances explicites : la communauté de pratique devient alors une communauté épistémique engagée dans la production de connaissances nouvelles (Haas, 1992). La frontière entre ces deux formes est en réalité très fine et il n'est pas rare de trouver au sein d'une même communauté en ligne des traits de chacune d'entre elles (Millerand et al. 2012). Communauté de pratique et communauté épistémique sont des idéaux-types, forment les deux faces d'une même pièce et peuvent être rassemblées sous l'appellation de communauté de savoir (Stassin, 2016).

Les savoirs qu'acquièrent les patientes sont qualifiés de "profanes" (Méadel, 2010) pour être distingués des savoirs "savants " et "experts " des médecins et des chercheures. Ils peuvent être rapprochés des savoirs "amateurs». Tout comme l'amateur de science, le patient expert « s'approprie des savoirs non académiques, n'est (en général) pas payé pour savoir et l'exercice de son savoir ne se fait pas lors de son temps de travail " (Jouet, Las Vergnas, 2010:5). Il s'agit d'« une "expertise ordinaire" acquise par l'expérience qui lui permet de réaliser, pendant son temps libre, des activités qu'il aime et qu'il a choisies »(Flichy, 2010 : 9). Ainsi, l'expertise qu'acquièrent les patient-es peut être très développée et régulièrement étoffée par des lectures et une veille informationnelle, par des participations actives et répétées à des communautés en ligne. Cette expertise peut dans certains cas se montrer complémentaire au savoir clinique du médecin (Akrich, Méadel, 2002), mais n'est cependant pas à mettre au même plan (Paganelli et al., 2014).

\section{Remédiation des savoirs et réappropriation de la parole trans par les trans}

13 Les membres de communautés de savoir évoluant dans le domaine de la santé acquièrent des compétences et des capacités d'agir (empowerment) qui les incitent à remettre en cause l'autorité des experts et des traditionnels gatekeepers dans la diffusion des savoirs et de l'information, qui les inscrivent dans une logique d'émancipation et les rendent autonomes face à leur santé. Par exemple, dans les années 1980-90, les malades du SIDA - et notamment les membres d'Act Up - et les victimes de catastrophes sanitaires (Tchernobyl, vache folle, amiante) se sont révélées expertes de leur maladie et/ou du problème qui les affectait, aptes à débattre avec les médecins et les responsables politiques et à s'imposer dans les débats publics (Bonneuil, Joly, 2013).

14 La remise en cause des experts est un élément essentiel du développement des savoirs sur la question trans par les personnes transgenres elles-mêmes. Par leur expérience de 
la transidentité (tant sur le plan médical, psychique que social), elles ont développé une expertise apte à rivaliser voire à contredire le savoir des médecins. Ce développement est incarné par ce qu'Arnaud Alessandrin (2018:60) nomme l'« explosion associative des années 2000 » qui va conduire à " une réappropriation des luttes et de la parole trans par les trans» (ibid. : 63). Par exemple, en France, les associations militantes comme Outrans dénoncent régulièrement l'hégémonie de la Sofect $^{1}$ dans la prise en charge des transitions et surtout « des années de maltraitance, de psychiatrisation systématique, d'examens gynécologiques intrusifs et inutiles, de pathologisation et d'infantilisation $»^{2}$.

15 Jusqu'en 2010, en France 3 , le «transsexualisme » (terme issu du vocabulaire médical) est considéré comme une affectation psychiatrique, un trouble mental que l'on pourrait "guérir » en " transformant " un homme en femme et une femme en homme par une chirurgie de réattribution sexuelle. Mais la transidentité n'est pas une question de «transformation» et est d'abord une question de genre avant d'être une question de sexe. L'opération de réattribution n'est donc pas le but de toutes les transitions. Les actes de chirurgie visent à féminiser ou masculiniser les corps, mais ne concernent pas systématiquement les organes génitaux. Certaines personnes n'ont en outre recours à aucun traitement hormonal ou chirurgical, d'autres sont non binaires et ne se reconnaissent dans aucun des deux genres ou au contraire se réclament des deux. La transidentité se vit donc de différentes manières. Le recours au terme " transgenre » en remplacement de "transsexuel» permet d'englober cette réalité, de dépasser l'approche "socio-corporelle» et binaire (Thomas, 2010). Ainsi, l'action des associations militantes vise, entre autres, à constituer un bien commun à destination des trans et de leurs alliées permettant de dépasser la médicalisation et la pathologisation des parcours longtemps imposées par les psychiatres (Bujon, Dourlens, 2012), contribuant au rejet des personnes, au sein de leur famille et de la société, et au fait que la transidentité soit encore associée, dans l'imaginaire collectif, à une « maladie mentale » (Reucher, 2015).

Enfin, le développement des savoirs trans favorise aussi l'autonomisation dans les parcours et dans les choix. Il s'échange dans les forums et les médias sociaux des noms de médecins jugés safe (en français : sûr) et bienveillants, des commentaires et des avis sur les chirurgien-nes, sur les endocrinologues qui acceptent de délivrer des hormones sans avis psychiatriques. Les réseaux informationnels instaurent une sorte de « marché des réputations " (Alessandrin, 2012), offrent la possibilité de réaliser une véritable «étude de marché » afin de choisir les bons interlocuteurs (médicaux et administratifs) et d'échapper aux lenteurs et aux lourdeurs du protocole hospitalier, notamment pour les personnes qui ont les moyens financiers de passer outre, en s'adressant à des cliniques privées et/ou étrangères (Beaubatie, 2020).

\section{Étude du site Wiki Trans : éléments de méthode}

Dans la continuité des travaux entrepris dans le champ francophone des sciences de l'information et de la communication par Mélanie Lallet et Lucie Delias, nous menons actuellement une étude dédiée aux pratiques informationnelles des personnes transgenres. Cette étude repose sur une participation à des réunions et groupes de parole organisés par une association LGBTQI du Grand Est, sur la réalisation d'entretiens avec des femmes et des hommes trans, dont la transition est en cours ou 
achevée, mais également sur le suivi, la «lecture » et l'observation de sites web et de comptes Twitter, Facebook, Instagram ou YouTube alimentés par des personnes trans reconnues comme influentes ou non-, que nous identifions en partie par le biais des entretiens. En effet, nous interrogeons les personnes sur leur usage d'Internet et des médias sociaux et cherchons également à savoir si elles produisent et publient des contenus numériques en lien avec leur transition, animent des groupes de paroles en ligne, etc. Nous nous plaçons donc dans une perspective ethnographique qui nous conduit à nous immerger dans les terrains entrepris, à analyser les dispositifs numériques mobilisés par les personnes trans et à les restituer dans leur contexte d'usage.

Au cours des entretiens, le site Wiki Trans a souvent été mentionné comme une source d'information. C'est un site dont nous ne connaissions pas l'existence avant le lancement de notre étude. La présence du terme «wiki » a rapidement suscité notre curiosité car il nous renvoyait au concept de communauté de savoir que nous avons mobilisé et développé dans de précédents travaux (Stassin, 2016), et plus particulièrement à des communautés de savoir regroupées autour de la technologie wiki telles que Wikipédia (Goldenberg, 2010), Tela Botanica (Heaton et al., 2011) ou encore GeoRezo (Jullien et al., 2010). Nous avons donc décidé de consacrer à ce site une étude à part entière. Outre le fait de susciter notre curiosité, la présence du terme «wiki » a également orienté notre méthodologie d'analyse en nous faisant pressentir d'emblée que la visée principale du site était informative et explicative, voire pédagogique, qu'il ne s'agissait pas d'un forum ou d'un groupe de discussion et que son fonctionnement reposait sur l'écriture collaborative et la mise en commun d'expériences et de connaissances sur un sujet précis : la transidentité et le parcours de transition. Du point de vue méthodologique, cette étude repose principalement sur deux approches: l'entretien et l'analyse du dispositif sociotechnique et infocommunicationnel que constitue le site.

Nous avons tout d'abord conduit un entretien avec la conceptrice et fondatrice du Wiki Trans : Menica Folden. L'enjeu était de comprendre les raisons qui l'ont poussée à ouvrir ce site, alors que le web semble déjà bien fourni en ressources documentaires liées à la transidentité et à la transition, d'accéder à l'imaginaire qui l'a guidée dans sa conception et dans l'anticipation des attentes et des usages du public et d'en savoir plus sur les modalités de participation et de contribution proposée aux membres de la communauté. Un second entretien mené auprès de $\mathrm{R}$, une personne membre de l'association où nous enquêtons, qui a rédigé un article pour le site et s'est servi de ce dernier pour fournir des informations à ses parents au moment de son coming out, nous a permis d'obtenir d'autres données relatives à son fonctionnement.

Ces entretiens ont été complétés et mis en perspective par une analyse du site. Pour cela, nous nous sommes appuyée sur différentes grilles d'analyse. La première, issue des travaux d'Orban de Xivry et al. (2007), propose de porter une attention à l'implication de l'énonciateur dans ses énoncés - implication qui peut être confondue (il est lui-même l'objet de l'énoncé) ou distanciée - et à l'interaction qu'il propose au public. Cette dernière peut être forte si le public est invité à réagir, prendre la parole, contribuer, ou bien faible, voire inexistante. L'analyse des degrés d'implication et d'interaction permet alors de définir le projet ${ }^{4}$ communicationnel d'un blog ou d'un site : informer ou partager des ressources, des connaissances; témoigner sur un vécu, une expérience ; faire part de ses sentiments et de ses états d'âme ; partager un point 
de vue, une opinion, lancer un débat, une polémique. La seconde grille, issue des travaux de Jouët et Le Caroff (2013), nous invite à analyser le cadre sociotechnique dans lequel le dispositif se déploie, c'est-à-dire ce qu'il permet au plan technique (architecture, charte graphique, outils de participation, de discussion, zone de commentaire, boutons de partage, etc.) et au plan social (captation de l'attention, Nétiquette, tactiques de visibilité, formes de contribution et d'échanges, modalités de circulation, etc.).

21 La troisième grille d'analyse a été bâtie par nos soins. Nous nous sommes appuyée sur nos précédents travaux dédiés aux communautés de savoir en ligne pour analyser la structure du « regroupement social » formé par les contributeur.trice's, leur degré de participation et de contribution, leurs règles de gouvernance et de bonnes conduites. Enfin, pour analyser le contenu thématique et discursif des ressources, nous nous sommes appuyée sur notre revue de la littérature des pratiques informationnelles des personnes transgenres puisqu'elle nous a permis d'identifier la nature des informations dont elles ont généralement besoin au cours de leur parcours de transition (témoignages, informations sur les traitements hormono-chirurgicaux, sur les démarches administratives, informations libérées du discours pathologisant et infantilisant des traditionnelles instances médicales, etc.).

\section{Projet info-communicationnel et fonctionnement du Wiki Trans}

\section{Centraliser des informations dispersées}

Wiki Trans a été créé en 2018 à l'initiative de Menica Folden. Tout commence sur les médias sociaux, en 2017, où elle poste, au cours de sa transition, des messages et des selfies. Suivie par cinq-cents puis mille personnes - et aujourd'hui près de six mille elle répond volontiers aux questions qu'on lui pose au sein de Twitter, mais surtout de Curious Cat. Sa visibilité et son nombre d'abonnés augmentant jour après jour, les sollicitations se font de plus en plus nombreuses: "J'étais bombardée de questions, je passais une heure par jour à y répondre» (entretien). Après un certain temps, elle se rend compte que les mêmes questions reviennent souvent et que ses réponses finissent par se perdre dans le flux des messages: "Je disais: "vas voir plus loin, j'ai répondu à cette question il y a deux mois". Et deux jours plus tard, quelqu'un d'autre me reposait la même question" (entretien). Selon elle, de nombreuses informations essentielles sont dispersées, voire cachées, à différents endroits du web: «Les ressources, elles se transmettent avant tout de bouche à oreille sur les réseaux sociaux et sur Discord et sur des chaînes YouTube. Mais dans une vidéo, l'info que tu cherches est parfois cachée dans un bout de trente secondes. L'info est donnée en trente secondes dans une vidéo de vingt minutes" (entretien). La dispersion des ressources qu'elle a pu observer n'est pas propre à la sphère francophone et a été mise en exergue par d'autres personnes transgenres interrogées dans le cadre d'autres études (Augustaitis et al., 2021).

La perte de visibilité des réponses qu'elle formule sur Curious Cat et l'identification d'un jeu de questions revenant régulièrement la pousse à les centraliser dans un seul et même endroit: d'abord un site sur WordPress, où chaque réponse donne lieu à un article, puis sur un wiki, qui lui permet de mieux structurer l'information et de se lancer dans un projet d'écriture collaborative avec d'autres personnes : "On peut appeler 
ça la méthode effectuale, comme en gestion de projet, je suis partie de ce que j'avais et j'ai construit à partir de là, en petites itérations. Je ne me suis pas dit, "je vais créer Wikipédia pour les trans", ce n'est pas ça qui s'est passé (rires)» (entretien). À travers le vocabulaire technique qu'elle emploie, on comprend que l'on a affaire à une personne très au fait de la gestion de projet numérique. En effet, elle est product designer de profession : «Mon job c'est de créer des produits, des sites web, des applications, [...] c'est de faire en sorte que l'information soit simple et accessible [...] je me suis dit qu'il y avait une opportunité de mettre mes compétences au service de ma communauté» (entretien). Wiki Trans est donc également né de la volonté d'associer un savoir expérientiel, profane et amateur relatif à la transition et un savoir-faire professionnel en développement web et gestion de l'information.

Bien qu'il ne s'agisse pas de créer une "Wikipédia pour les trans", il s'agit de centraliser des informations dispersées, mais aussi de traduire des ressources produites en langue anglaise ou encore de vulgariser de l'information scientifique : "On reformule pour que ce soit intelligible et lisible. Il y a un gros enjeu de reformulation du contenu et de restructuration de l'information en plein de petits articles» (entretien). Le site se présente comme « un portail d'information à destination des personnes trans en questionnement ou en cours de transition, [de] leurs proches et leurs alliés» (extrait du Manifeste du Wiki Trans ${ }^{5}$ ). Il vise à faciliter les recherches d'information des personnes trans, et ce, quelle que soit l'étape à laquelle elles se trouvent, mais également des cisgenres dont les besoins informationnels peuvent émerger lorsqu'une de leurs proches fait son coming out: "Nous voulons que toutes les informations nécessaires pour chaque type de besoin soient disponibles, vérifiables, et à disposition de manière centralisée " (extrait du Manifeste).

\section{Une écriture et une mise en commun encadrées et hiérarchisées}

Le fait que le site se donne pour mission de vulgariser de l'information scientifique, mais aussi de produire des contenus en lien avec des traitements médicaux et chirurgicaux a poussé sa fondatrice à redoubler de vigilance quant aux modalités de contribution, d'éditorialisation et de publication. Ses compétences professionnelles jouent de toute évidence un rôle important dans la gestion et la construction du site et dans la manière dont il répond aux besoins informationnels identifiés dans la communauté. Contrairement à Wikipédia, au sein de laquelle tout un chacun peut rédiger ou modifier un article et où les contenus sont validés a posteriori, les contributions sur Wiki Trans sont très encadrées. Certes, tout le monde peut contribuer, mais les propositions d'articles doivent être préalablement soumises à l'équipe de rédaction qui se charge de la vérification et de la validation avant publication. De même, les articles ne peuvent pas être directement modifiés sur le site, mais un onglet «nous contacter " à la fin de chacun d'entre eux permet d'écrire à l'équipe pour toute question ou tout commentaire. Wiki Trans a en outre un fonctionnement de type hiérarchique, qui est propre à de nombreuses communautés en ligne et notamment aux communautés orientées vers le partage et/ou la production de connaissances. L'équipe de rédaction forme le noyau dur qui décide des sujets à aborder, valide les articles et se réserve le droit d'exclure les membres qui ne respectent pas les valeurs et les règles de bonnes conduites exposées dans le Manifeste : "Nous voulons le meilleur pour les personnes trans et pour la communauté trans, féministe et LGBT. Nous donnons l'exemple en donnant le meilleur de nous-mêmes, dans un état d'esprit collaboratif, communicatif, empathique et constructif ». 
L'équipe de rédaction est principalement composée de Menica Folden, de Tieffeline et de Julie T. qui se sont - comme nous le verrons plus loin - entourées d'expert-es pour s'assurer de la fiabilité des informations publiées en ce qui concerne notamment le champ médical. Toutes trois sont bénévoles, mais ont à cœur de travailler avec soin : "Nous nous engageons aussi à concevoir du contenu de qualité, vérifié et sourcé", (extrait du Manifeste). On retrouve ici une caractéristique des amateures engagées dans la publication de contenus culturels ou scientifiques déjà mise en avant dans la littérature scientifique, à savoir le fait qu'ilselles investissent dans leurs productions « un même sens de l'obligation, une même anxiété, une même exigence de résultat que dans l'univers professionnel, [...] refusent l'étiquette jugée péjorative de l'amateurisme et souhaitent que leur engagement bénévole soit évalué selon des standards professionnels" (Cardon et DelaunayTéterel, $2006: 53)$.

L'écologie informationnelle de Wiki Trans repose sur différents outils. Les échanges et discussions concernant les articles se font au sein d'un espace de discussion ouvert sur Discord et non pas au sein du wiki. Les nouveaux contenus sont signalés et mis en valeur sur Twitter via le compte @wiki_trans, ou via les comptes personnels des membres de l'équipe de rédaction. Mais Twitter est également utilisé pour des appels à contribution, comme nous le relate $\mathrm{R}$ :

«J'étais sur Twitter et j'ai vu un appel de Menica Folden qui cherchait des informations sur les transitions administratives en milieu scolaire et universitaire. Donc je lui ai répondu que j'avais beaucoup d'infos dans le scolaire et un peu dans l'universitaire ayant été quelques fois confrontée à la démarche que ce soit à titre personnel ou pour des connaissances. Dans un premier temps j'ai fait un thread sur Twitter puis on l'a transposé dans un Google doc pour compléter les informations avec des articles de lois, les différents règlements et circulaires ministérielles » (entretien).

Les contributions se font donc selon deux modes: le mode push au sens où une personne propose spontanément un sujet ou un article à l'équipe de rédaction, le mode pull ou l'on va chercher des contributeurtrice's sur les médias sociaux. Enfin, Twitter est également utilisé pour sonder le public sur ses nouveaux besoins informationnels (figure 1).

Wiki Trans

@wiki_trans

\title{
Petite question en passant : est-ce qu'il manque des trucs sur le site pour les personnes en questionnement? Des choses que vous avez vu ailleurs qui vous auraient aidé ?
}

\author{
12:43 PM · 13 févr. 2021 - Twitter for Android
}

Figure1. Tweet de Wiki Trans sondant les besoins informationnels du public 


\section{Des ressources pour faciliter la transition sociale et administrative}

29 La transition est un processus social souvent complexe et long, empreint d'incertitudes et de craintes. Une fois l'étape du questionnement passée vient celle du coming out à ses proches, à ses camarades de classe ou à ses collègues de travail. Les stratégies de révélation diffèrent d'une personne à l'autre : certaines font leur coming out avant d'entamer leur transition, d'autres préfèrent attendre qu'elle soit bien avancée pour le faire. D'autres encore cherchent à collecter des documents qui vont venir en appui à leurs «explications». Les réactions de l'entourage varient également, allant d'un soutien sans faille à un rejet brutal et total (Brumbaugh-Johnson, Hull, 2019).

Pour aider les personnes trans à préparer au mieux cette transition, mais aussi les personnes cis à accepter et comprendre la transition de leur proche, le site met à disposition de nombreuses ressources sous forme d'articles (d'environ 5000 signes), de foires aux questions avec des réponses courtes, ou encore de guides et de brochures à télécharger. Il regorge aussi d'informations en lien avec les démarches administratives (modèles de lettres et articles explicatifs sur le changement d'état civil et de numéro de sécurité sociale, etc.) et médicales (lien vers des bases de données recensant psychiatres, endocrinologues, orthophonistes jugés safe par la communauté) qui peuvent autant intéresser les personnes trans que leurs parents, notamment lorsqu'elles sont mineures. En outre, un lexique répertoriant de nombreux termes liés à la transidentité offre des repères sémantiques qui peuvent être complétés ou approfondis par la lecture de sites de linguistique signalés par des liens hypertextes.

Certaines ressources sont clairement adressées aux personnes trans, comme les articles dédiés au passing, c'est-à-dire au fait de réussir à " passer pour » une personne cisgenre en société, de pouvoir être identifié directement par autrui au bon genre. Si cela passe par un traitement hormono-chirurgical, cela passe aussi par un travail sur sa posture, la manière de se coiffer ou de se vêtir, de poser sa voix, etc. De nature prescriptive, ces articles délivrent conseils et instructions, montrent les différentes étapes pour faire telle ou telle chose en appui sur des photos, des schémas, des vidéos tutorielles que l'on peut aussi retrouver sur YouTube. Notons cependant que le rapport au passing n'est pas le même selon les personnes. Si certaines cherchent à se fondre dans la masse, d'autres refusent la catégorisation binaire homme/femme, revendiquent la visibilité à des fins notamment militantes ou politiques (Beaubatie, 2019). Un homme trans (FtoM) cherchera ainsi à effacer au mieux les cicatrices d'une mammectomie quand un autre les cultivera comme symbole "d'une libération au sens de fierté et d'émancipation » (Espineira, 2018).

Le site étant doté d'une rubrique «Pour les alliés", d'autres ressources sont, elles, destinées aux personnes cis. Des articles explicatifs ou prescriptifs, de type «Comment me comporter face à une personne trans?" ou "Petit recueil des phrases à éviter ", permettent de découvrir les questions ou les sujets à éviter afin de ne pas blesser ou importuner. Différentes brochures ont également vocation à être remises aux familles ou aux employeurs. $\mathrm{R}$ qui était lectrice du site avant d'en être contributrice en a par exemple envoyé une à ses parents : "Je leur ai envoyé la brochure "Dix idées reçues sur la transidentité" qui est bien faite et surtout bien sourcée parce qu'il y avait quelques petits doutes de leur part et que je voulais, entre guillemets, leur remettre les points sur les i sur le sujet de la transidentité. [...] Ils en ont fait une très bonne réception » (entretien). 
33 Le site permet également aux personnes trans et à leurs proches de témoigner, de raconter leur parcours par le biais d'une rubrique dédiée : «Parce qu'il est impossible de se projeter dans quelque chose qu'on ne connait pas, on ne peut pas envisager une transition sans savoir que ça nous est accessible", (extrait de la rubrique "Témoignages " ${ }^{6}$ ). Les contributeur.trice's sont invitées à répondre à différentes questions posées dans un formulaire qui va alors faciliter le schéma narratif du témoignage, mais surtout orienter le récit pour mettre en avant les éléments positifs de la transition. Pour la fondatrice, cela est essentiel, car si certains parcours sont marqués par des moments difficiles, anxiogènes et dépressogènes, d'autres sont aussi une véritable libération et conduisent vers un état d'épanouissement personnel, un aspect qu'elle souhaite mettre en valeur. Dans la même lignée, une rubrique "Trombinoscope " répertorie de nombreuses personnalités publiques : "il est important de voir que des personnes trans ont réussi, avant nous, à commencer leur transition et réussir dans leur vie, de surcroît dans l'espace public (auteur, avocat, député, journaliste, homme ou femme d'affaires...). Cette page leur rend hommage, et vous permet de voir que oui, être trans et envisager son futur de manière positive, c'est possible $»^{7}$. Si la formulation peut sembler maladroite, car laissant entendre que la réussite passe par la célébrité, il faut plutôt la comprendre comme une volonté de montrer que ce sont des personnes qui s'assument et s'exposent ouvertement aux regards des autres de façon régulière et intense, donc que l'on peut prendre pour modèle. Il faut aussi l'envisager comme un moyen de donner une image positive de la transidentité, de dépasser les discours pathologisants de certains médecins et les stéréotypes négatifs véhiculés par les médias, au sein desquels les personnes trans sont peu représentées, sont souvent associées à la fragilité, l'instabilité, le travestissement, la prostitution, et interrogées sur leur "changement de sexe", voire de sexualité, renforçant l'idée reçue que genre et sexe fonctionnent de pairs, et que transition signifie nécessairement changement d'orientation sexuelle. Comme le souligne Karine Espineira (2015 : 208) : "[l]'inventaire des déficits en représentation, en médiation donne la mesure du phénomène: déficits d'ancêtres, de mémoires, d'histoires, de théories, de sources, de récits non-binarisants et non-hétérosexualisants".

\section{Vulgarisation de l'information liée à la transition médicale}

Le parcours de transition peut aussi être un parcours médical basé sur un traitement hormonal substitutif (THS) et sur des actes chirurgicaux féminisants ou masculinisants. Comme toute prise de médicaments, la prise d'hormones n'est pas anodine et n'est pas sans conséquence sur l'organisme. Les effets sont différents d'un organisme à l'autre. Le suivi médical est important puisqu'il permet de déceler les contre-indications et d'adapter les dosages en fonction de la physiologie de chacun'e. C'est d'ailleurs pour cela que les associations déconseillent l'automédication ou l'achat d'hormones sur Internet.

Avant de commencer leur traitement ou en tout début de traitement, les personnes ont pour la plupart à cœur d'en comprendre le fonctionnement et les effets. Cela nécessite une importante phase de lecture et une familiarisation, pas toujours évidente, avec le vocabulaire médical et scientifique. Comme nous l'avons vu plus haut, le manque de ressources documentaires en langue maternelle et la non-accessibilité (intellectuelle) $\mathrm{du}$ discours scientifique peuvent être un frein à la recherche d'information et à la satisfaction du besoin informationnel, donc un potentiel frein à la transition. Un autre objectif du Wiki Trans est donc de proposer des articles qui expliquent de manière 
simple et efficace des aspects complexes des traitements hormonaux (fonctionnement, effets sur le corps et le moral, effets secondaires) et des opérations chirurgicales (objectifs, étapes, conséquences, résultats, convalescence), des articles qui pour la plupart relèvent de la vulgarisation scientifique.

Les articles sont présentés selon deux formats. On relève tout d'abord un format court (600 à 1000 signes) intégré dans des foires aux questions et apportant une réponse synthétique à une question qui peut être complexe ("Que se passe-t-il en cas d'arrêt du traitement? », «Quels effets sur ma fertilité ? ») ${ }^{8}$. D'autres articles ont un format plus long (6000 signes) et apportent des réponses plus détaillées. On y décèle une visée pédagogique et une simplification du discours qui passent par l'emploi de métaphore ( les hormones sont des clés qui ouvrent des serrures (les récepteurs)») ou bien par l'intégration de schémas pour expliquer, par exemple, l'arrêt naturel de la production d'hormones sexuelles par le corps avec la prise du $\mathrm{THS}^{9}$ ou bien les différentes étapes d'une laryngoplastie (chirurgie de la pomme d'Adam) ${ }^{10}$.

Enfin, dans une perspective de fiabilité, de pertinence et de crédibilité, les articles intègrent les sources à partir desquelles ils ont été écrits, soit sous forme de liens hypertextes dans le corps du texte, soit sous forme de «bibliographie » en fin d'article. L'analyse de ces sources permet de les répartir en quatre catégories :

- Les sites de praticiennes (psychologues, psychiatres, chirurgiennes), qui sont pour la plupart anglophones;

- Les sites des associations transgenres (Espace Santé Trans, OUTrans, ACCEPTESS-T), qui jouent un rôle historique et important dans la vulgarisation des traitements médicaux et de l'information scientifique ;

- Les bases de données publiques des médicaments telles que Vidal.fr ou la base de l'Agence nationale de sécurité du médicament et des produits de santé (ANSM) permettant d'accéder à des données et documents de référence sur les médicaments commercialisés ou ayant été commercialisés au cours des dernières années ;

- Les articles scientifiques, en langue anglaise ou française, publiés en revue et accessibles en libre accès sur des plateformes comme OpenEdition ou ScienceDirect ou encore sur des carnets de recherche de la plateforme Hypothèses.

Les contributeur.trice's s'appuient donc en partie sur des ressources issues des bases de données médicales en ligne et des articles issus de revues académiques en libre accès qui offrent «de nouvelles possibilités informatives et cognitives» (Méadel, $2010: 111$ ) et suscitent une forme de "trivialité ». Cette trivialité, qu'Yves Jeanneret définit comme «le caractère fondamental des processus qui permettent le partage, la transformation, l'appropriation des objets et des savoirs au sein d'un espace social hétérogène » (Jeanneret, 2008 : 20), émane ici de la réécriture, de la traduction, de la vulgarisation donc de la reformulation des ressources scientifiques. Ces dernières sortent alors de la sphère académique pour devenir accessibles tant physiquement (sur le wiki) qu'intellectuellement (car vulgarisée) au grand public ou tout au moins au public du wiki.

\section{Hybridation au sein d'une communauté de savoir}

39 Le recours au concept de "communauté de savoir " s'avère opérant pour l'étude et l'analyse d'un ensemble de personnes regroupées autour d'un intérêt commun pour un domaine particulier et dont le projet vise le partage et/ou la production de 
connaissances en lien avec ce domaine. Au sein de ce type de communauté, les processus cognitifs revêtent une dimension sociale, située et distribuée (Suchman, 1987; Hutchins, 1995; Conein, 2004). Sociale parce que les connaissances sont construites à travers les échanges et les interactions entre individus. Située, parce que l'apprentissage et l'échange de connaissances sont ancrés dans le contexte dans lequel ils s'opèrent (ici le parcours de transition). Distribuée parce que les membres sont liées par une interdépendance cognitive, au sens où ils·elles produisent et acquièrent tour à tour des connaissances et que ces dernières ne sont ni produites individuellement ni détenues totalement par un individu. La réciprocité est un élément important puisqu'il renvoie directement à l'étymologie de la communitas ${ }^{11}$ et aux logiques de don (munus) et de contre-don qui traversent tout regroupement communautaire. Chaque membre détient donc des compétences et des connaissances dont les autres ont besoin pour réaliser une tâche commune, apporte sa pierre à l'édifice en se positionnant sur les thématiques avec lesquelles ileelle est à l'aise. Menica Folden, qui, outre le fait de mettre ses compétences professionnelles à disposition du projet, se charge par exemple des rubriques liées au social et à l'administratif, quand Julie T. signe la plupart des articles médicaux. Cette dernière n'est pas médecin, mais membre du Réseau de Santé Trans (ReST) qui associe des personnes trans, des représantes d'associations, et des professionnelles de santé en région Bretagne. À travers son parcours elle a développé un intérêt et une expertise pour les aspects médicaux.

Le profil des membres se caractérise alors par une forte hétérogénéité. On compte des personnes trans (et cis, notamment dans les témoignages) qui mettent leurs vécus et savoirs expérientiels, profanes et amateurs au service de la communauté, mais on compte aussi des professionnel-les et des médecins du ReST auxquels sont soumis, pour validation, les contenus médicaux et scientifiques. Ce pont entre Wiki Trans et le ReST est notamment construit par le biais de Julie T. qui forme ce que l'on nomme dans la théorie des communautés de pratique une "actrice interface" capable de faire dialoguer des mondes sociaux et des milieux hétérogènes - ici les médecins et les bénévoles -, contribuant alors à la création de nouvelles significations (Wenger, 1998). Enfin, l'équipe de rédaction prend en compte les avis et les remarques que peuvent leur faire certaines associations trans également spécialistes de la vulgarisation des connaissances en lien avec les traitements hormonaux et peut être amenée à modifier ou enrichir les contenus en fonction de ces avis ou remarques. Cette communauté de savoir se caractérise alors par une forte « hybridation » (Boure, Lefebvre, 2015) entre savoirs expérientiels, profanes et amateurs et savoirs médicaux et scientifiques.

\section{Conclusion}

41 L'hétérogénéité des profils des contributeur-trice·s donne donc lieu à la constitution de savoirs hybrides, mais aboutit aussi à la production d'un corpus de ressources si ce n'est «hybride» tout au moins «mosaïque». Le terme «mosaïque», que nous empruntons à Viviane Couzinet (2001), désigne un " ensemble d'éléments disparates » et met en exergue le fait que les ressources de ce "corpus " sont de natures diverses (articles, brochures à télécharger, questions-réponses, etc.) et ont différentes visées communicationnelles. On relève une visée majoritairement informative et explicative perceptible dans les contenus médicaux vulgarisés. Cette visée explicative se retrouve aussi dans les ressources ou les brochures à destination des personnes cisgenres (les 
proches, les parents, les employeurs), mais se double alors d'une dimension prescriptive (ce qu'il faut faire et ne pas faire, ce qu'il faut dire et ne pas dire) relevant du «savoir-être ». Cette dimension prescriptive se retrouve aussi dans les contenus dédiés à la transition administrative et sociale ou dans les articles abordant le passing, faisant véritablement office de tutoriels et relevant plutôt ici du «savoir-faire ». Enfin, la rubrique "témoignage » accueille une diversité de textes, forme une zone narrative au sein de laquelle le «nous » se fragmente en une multiplicité de « je » venus raconter leur parcours ou celui de leur fille, de leur fils, de leur adelphe, de leur ami-e. À cela s'ajoute une galerie de portraits photographiques représentant des célébrités, femmes, hommes, personnes non binaires visant à combler ce que Karine Espineira (2015) nomme le « déficit de représentations ».

Les projets info-communicationnels du Wiki Trans sont donc multiples, mais convergent vers un projet principal : proposer un lieu de centralisation des ressources, un lieu facilitant les recherches d'information, mais un lieu qui peut aussi être le point de départ vers de nouvelles recherches. En effet, les nombreuses références bibliographiques et liens hypertextes qui parsèment les ressources et garantissent leur fiabilité sont autant d'invitations à poursuivre la lecture, à compléter ou approfondir "ailleurs" les nombreux points abordés «ici ». Plus qu'un site, Wiki Trans est un dispositif sociotechnique et info-communicationnel reposant sur un fonctionnement réticulaire (réseau d'individus, réseau de liens hypertextes, réseau de documents) et communautaire, ayant la particularité d'être très encadré par une équipe de rédaction qui reste attentive à ce que la ligne éditoriale qu'elle s'est fixée soit respectée, mais qui n'est pas pour autant repliée sur elle-même, qui se tourne vers l'extérieur. Il reste désormais à analyser la place qu'occupe Wiki Trans dans l'écologie informationnelle de la transidentité ainsi que la réception et l'usage qu'en font réellement les publics. On peut tout à fait imaginer que la vulgarisation des informations médicales liées aux traitements hormonaux satisfasse tout autant le besoin informationnel d'une femme ou d'un homme trans que celui d'une personne cisgenre devant elle aussi, pour des raisons de santé, prendre ce type de traitements. Cela ouvre donc la voie à de nouvelles perspectives de recherche.

\section{Bibliographie}

Adams, S. \& Peirce K. (2006) Is There a Transgender Canon?: Information Seeking and Use in the Transgender Community. Proceedings of the Annual Conference of CAIS / Actes Du Congrès Annuel de l'ACSI. https://doi.org/10.29173/cais161.

Akrich, M. \& Méadel, C. (2002). Prendre ses médicaments/prendre la parole : Les usages des médicaments par les patients dans les listes de discussion électroniques. Sciences Sociales et Santé, 20(1), 89-116. https://doi.org/10.3406/sosan.2002.1546 
Alessandrin A. (2012) Du 'transsexualisme' aux devenirs Trans (Thèse de doctorat en sociologie, Université Bordeaux Segalen)

Alessandrin, A. (2018). Sociologie des transidentités. Paris : Cavalier Bleu Eds.

Augustaitis, L., Leland M., Gamarel, K. \& Haimson, O (2021). Online Transgender Health Information Seeking : Facilitators, Barriers, and Future Directions. CHI'21,May 8-13,2021,Yokohama, Japan. http:// oliverhaimson.com/PDFs/AugustaitisOnlineTransHealthInfo.pdf

Beaubatie, E. (2019). L'aménagement du placard. Geneses, 1(114), 32-52. https://doi.org/10.3917/ gen.114.0032

Beaubatie, E. (2020). Transfuges de sexe. Genre, santé et sexualité dans les parcours d'hommes et de femmes trans' en France (Thèse de doctorat en sociologie, École des Hautes Études en Sciences Sociales)

Bonneuil, C. \& Joly, P. B. (2013). Sciences, techniques et société. Paris : Éditions La Découverte.

Boure, R. \& Lefebvre, M. (2015). Entre science légitime et science amateur : le devenir trivial d'une information scientifique sur Internet. Les Enjeux de l'Information et de la Communication, $n^{\circ}$ 16, 143 à 152. https://lesenjeux.univ-grenoble-alpes.fr/2015/supplement-a/12-entre-sciencelegitime-et-science-amateur-le-devenir-trivial-dune-information-scientifique-sur-internet/

Bujon, T. \& Dourlens C (2012). Entre médicalisation et dépathologisation : la trajectoire incertaine de la question trans. Sciences sociales et santé 30, n 3, 33-58. https://doi.org/10.3917/sss.303.0033

Brumbaugh-Johnson, S. M., \& Hull, K. E. (2019). Coming Out as Transgender : Navigating the Social Implications of a Transgender Identity. Journal of Homosexuality, 66(8), 1148-1177. https://doi.org/10.1080/00918369.2018.1493253

Cardon, D. \& Delaunay-Téterel, H. (2006). La production de soi comme technique relationnelle. Réseaux, 138(4), 15-71.

Chaudiron, S. \& Ihadjadene, M. (2010). De la recherche de l'information aux pratiques informationnelles. Études de communication. Langages, information, médiations, $\mathrm{n}^{\circ} 35,13-30$. https:// doi.org/10.4000/edc.2257

Choo, C. W. (2006). The knowing organization : How organizations use information to construct meaning, create knowledge, and make decisions. Oxford : Oxford University Press.

Conein, B. (2004). Cognition Distribuée, Groupe social et Technologie cognitives. Réseaux, 124. https://doi.org/10.3917/res.124.0053

Couzinet, V., (2001). Médiations hybrides : le documentaliste et le chercheur en Sciences de l'information. Paris : ADBS Éditions.

Delias, L. \& Lallet M. (2018). La remédiation des savoirs en santé dans les communautés en ligne sur les transidentités. Revue française des sciences de l'information et de la communication, $\mathrm{n}^{\circ} 15$. https://doi.org/10.4000/rfsic.4813.

Deutsch, M. B. (2016). Evaluation of Patient-Oriented, Internet-Based Information on GenderAffirming Hormone Treatments. LGBT Health 3, $\mathrm{n}^{\circ} 3$, 200-207. https://doi.org/10.1089/lgbt. 2015.0116 .

Dupagne, D. (2011). E-santé. Communications, 88(1), 57-65.

Esposito, R., 2000. Communitas : origine et destin de la communauté. Paris : Presses universitaires de France. 
Espineira, K. (2015). Médiacultures. La transidentité en télévision. Une recherche menée sur un corpus à l'INA (1946-2010). Paris : L'Harmattan.

Espineira, K. (2018). Les corps trans : Disciplinés, militants, esthétiques, subversifs. Revue des sciences sociales, 59, 84-95. https://doi.org/10.4000/revss.701

Flichy, P. (2010). Le Sacre de l'amateur : Sociologie des passions ordinaires à l'ère numérique. Le Seuil.

Goldenberg A., (2010). La participation dans les communautés épistémiques : don ou contribution ? in : Millerand F., Proulx S., Rueff J. (dir), Web social : mutation de la communication (p. 217-230). Québec : Presses de l'université du Québec.

Granier, J. (2011). Du Contrat de Lecture au Contrat de Conversation. Communication \& langages, 3(3), 51-62. https://doi.org/10.4074/S033615001100305X

Haas, P. M. (1992). Introduction : Epistemic Communities and International Policy Coordination. International Organization, 46(1), 1-35.

Hawkins B. \& Gieseking J. (2017). Seeking ways to our transgender bodies, by ourselves : Rationalizing transgender-specific health information behaviors. in : Proceedings of the Association for Information Science and Technology 54, 1 (Jan. 2017), 702-704. https://doi.org/10.1002/

pra2.2017.145054011e22

Hawkins, B. \& Haimson, O. (2018). Building an Online Community of Care : Tumblr Use by Transgender Individuals. in : Proceedings of the 4th Conference on Gender \& IT (GenderIT '18). ACM, New York, NY, USA, 75-77. https://doi.org/10.1145/3196839.3196853

Heaton, L., Millerand, F. \& Proulx, S. (2011). Émergence d'une communauté épistémique : création et partage du savoir botanique en réseau. in : Proulx S., Klein A. (dir.), Connexions : communication numérique et lien social (p. 254-268). Namur : Presses universitaires de Namur.

Hutchins, E. (1995). Cognition in the Wild. Cambridge (MA) : MIT Press.

Jeanneret, Y. (2008). Penser la trivialité. vol. 1, La vie triviale des êtres culturels. Paris : HermèsLavoisier.

Jouet, E., \& Las Vergnas, O. (2011). Les savoirs des malades peuvent-ils être tenus pour des savoirs amateurs ? Alliage, 69. http://revel.unice.fr/alliage/index.html?id=3268

Jouët, J. \& Le Caroff, C. (2013). L'observation ethnographique en ligne. in : Barats C. (dir.), Manuel d'analyse du Web en Sciences Humaines et Sociales (p. 147-160). Paris : Armand Colin.

Jullien, N., Roudaut, K. \& Le Squin, S. (2010). Involving Oneself in an On-Line Community of Practice : The Case GeoRezo. SSRN Scholarly Paper. Rochester, NY : Social Science Research Network, 7 septembre 2010. https://papers.ssrn.com/abstract $=1748523$.

Karami, A., Webb, F. \& Kitzie, V. L. (2018). Characterizing Transgender Health Issues in Twitter. in : Proceedings of the Association for Information Science and Technology.

Kuhlthau, C. (1991). Inside the Search Process : Information Seeking from the User's Perspective. Journal of the American Society for Information Science, 42(5), 361-371

Lallet, M. \& Delias L. (2018) Les réseaux sociaux numériques et le développement controversé de savoirs d'expérience sur les transidentités. Le Temps des médias 31(2),137-55. https://doi.org/ 10.3917/tdm.031.0137.

Le Coadic, Y.-F. (2004). La science de l'information. Paris : Presses Universitaires de France.

Legros, M. (2009). Étude exploratoire sur les blogs personnels santé et maladie. Santé Publique, 21(hs2), 41-51. 
Méadel, C. (2010). Les savoirs profanes et l'intelligence du Web. Hermès, La Revue, 2(2), 111-117. https://doi.org/10.4267/2042/38647

Millerand, F., Heaton, L., \& Proulx, S. (2012). Émergence d'une communauté épistémique : Création et partage du savoir botanique en réseau. In : Proulx S. \& A. Klein, Connexions. Communication numérique et lien social (p. 253-268). Presses universitaires de Namur.

Orban de Xivry, A. C., Matagne, J. \& Klein, A. (2007). Typologie dynamique : une blogosphère de projets. In : Klein A. (dir.), Objectif Blogs ! Explorations dynamiques de la blogosphère (p. 37-68). Paris : L'Harmattan.

Paganelli, C, \& Clavier, V. (2011). Le forum de discussion : une ressource informationnelle hybride entre information grand public et information spécialisée. In : Yasri-Labrique E. (dir.), Les forums de discussion: agoras du XXIe siècle ? Théories, enjeux et pratiques discursives (p. 39-55). L'Harmattan.

Paganelli, C., Clavier, V. \& Duetto, A. (2014). S'informer via des médias sociaux de santé : quelle place pour les experts ?. Le Temps des médias, 2(2), 141-143. https://doi.org/10.3917/tdm.023.0141

Pohjanen, A. M. \& Terttu, A. M. K. (2016). Transgender information behaviour. Journal of Documentation 72(1) : 172-90. https://doi.org/10.1108/JD-04-2015-0043.

Reucher, T. (2005). Quand les trans deviennent experts. Multitudes, 20(1), 159-164.

Stassin, B. (2016). La blogosphère info-doc : une communauté de savoir, une mosaïque de médiations. Toulouse, France : Cépaduès-Éditions.

Saracevic, T. (1996). Modeling interaction in Information Retrieval (IR) : A review and proposal. in : Proceedings of the Annual Meeting of the American Society for Information Science, 33, 3-9. https:// www.researchgate.net/publication/ 239054075_Modeling_interaction_in_information_retrieval_IR_A_review_and_proposal Simonnot, B. (2006). Le besoin d'information : Principes et compétences. Thémat'IC 2006, Information : besoins et usages, 40-56.

Suchman, L. (1987). Plans and situated actions : The problem of human-machine communication. Cambridge : Cambridge University Presse.

Thomas, M-Y.(2010). De la question trans aux savoirs trans, un itinéraire. Le sujet dans la cité, 1, 120-29.

Wenger, E. (1998). Communities of practice : Learning, meaning, and identity. Cambridge : Cambridge University Press.

\section{NOTES}

1. La Société française d'études et de prise en charge de la transidentité réunit les professionnelles impliquées dans la prise en charge des personnes transgenres, dans des buts d'aide à la transition, de recherche et de formation. Ses méthodes sont jugées pathologisantes et médicalisantes et son hégémonie est contestée par les personnes trans et les associations. En 2019, elle devient la FPATH (French Professional Association for Transgender Health) en référence à la World Professional Association for Transgender Health. Mais pour les associations militantes, cela n'est qu'une opération de communication qui ne mettra pas fin à la psychiatrisation des parcours qu'elle impose.

2. Source: https://outrans.org/2020/12/08/lettre-ouverte-des-associations-trans-a-nicolasmorel-journel-president-de-la-sofect-et-de-la-fpath/

3. Décret du ministère de la Santé du 10 février 2010 publié au Journal officiel. 
4. Le terme "projet» est préféré par les auteures à celui de «contrat de lecture » ou de «promesse » que propose François Jost pour les émissions de télévision. Il permet de mettre en exergue le fait que le projet d'un blog peut être multiple ou changeant. Il est à rapprocher du «contrat de conversation» dont parle Jean-Maxence Granier (2011). Pour notre part, nous préférons le terme "projet info-communicationnel» pour renvoyer au fait que les médias sociaux sont à la fois des dispositifs de production de l'information, des dispositifs d'accès à l'information, des dispositifs de diffusion (communication) de l'information, donc des dispositifs info-communicationnel (Stassin, 2016).

5. Disponible sur : https://wikitrans.co/manifeste/

6. Voir : https://wikitrans.co/temoignages/

7. Voir : https://wikitrans.co/modeles/

8. Voir : https://wikitrans.co/ths/fem/faq/

9. Voir : https://wikitrans.co/ths/comment-fonctionnent-les-hormones/

10. Voir : https://wikitrans.co/2020/01/06/chirurgie-de-la-pomme-dadam/

11. La communitas est de l'ordre du communis, de ce qui est commun, du bien collectif et du bien public. Le terme communis est, quant à lui, formé à partir du rapprochement de la préposition cum et du nom commun munus. Le cum, qui signifie " avec ", implique la relation à l'autre et le vivre ensemble et le munus renvoie, quant à lui, au devoir, au service rendu, à l'obligation et au don. À la différence du « don » exprimé par le vocable donum (cadeau, présent, offrande, don), le « don » exprimé par le vocable munus revêt un caractère obligatoire (Esposito, 2000).

\section{RÉSUMÉS}

Cet article porte sur Wiki Trans, un site collaboratif créé par une femme transgenre pour permettre la mise en commun d'expériences et le partage d'informations liées aux aspects sociaux et médicaux de la transidentité et répondre aux besoins informationnels qu'elle suscite. Le site vise à centraliser des informations dispersées au sein du web, à traduire en français des ressources anglophones et à vulgariser de l'information médicale et scientifique. Ce projet favorise l'émergence d'une communauté de savoir en ligne composée de personnes transgenres et cisgenres, de bénévoles et de médecins. Une hybridation entre savoirs expérientiels et savoirs experts est alors observée.

This article deals with Wiki Trans, a collaborative website created by a transgender woman to pool experiences and share information related to the social and medical aspects of transidentity and to respond to the information need it raises. The site aims to centralize information that is scattered throughout the web, to translate English resources into French and to popularize medical and scientific information. This project promotes the emergence of an online knowledge community composed of transgender and cisgender people, volunteers and physicians. Hybridization between experiential knowledge and expert knowledge are then observed.

Dieser Artikel untersucht Wiki Trans, eine kollaborative Website, die von einer Transgender-Frau erstellt wurde, um Erfahrungen zu bündeln, Informationen zu den sozialen und medizinischen Aspekten der Trans-Identität auszutauschen und auf die dadurch entstehenden Informationsbedürfnisse zu reagieren. Ziel der Webpräsenz ist es, die im Web verstreuten Informationen $\mathrm{zu}$ zentralisieren, englische Texte ins Französische $\mathrm{zu}$ übersetzen und 
medizinische und wissenschaftliche Informationen allgemein bekannt und verständlich $\mathrm{zu}$ machen. Dieses Projekt fördert die Entstehung einer Online-Wissensgemeinschaft, die sich aus transgender und cisgender Personen, Freiwilligen und Ärzten zusammensetzt. Es wird eine Hybridisierung zwischen Erfahrungswissen und Expertenwissen beobachtet.

\section{INDEX}

Mots-clés : besoin informationnel, communauté de savoir, savoir expérientiel, transidentité Keywords : experiential knowledge, information need, knowledge community, transidentity Schlüsselwörter : Informationsbedarf, Wissensgemeinschaft, Erfahrungswissen, Trans-Identität

\section{AUTEUR}

\section{BÉRENGÈRE STASSIN}

Centre de recherche sur les médiations - Université de Lorraine 